\title{
Performance Enhancement of DS-CDMA System using Extended BPSK Modulation Scheme
}

\section{BARKHA PANDEY ${ }^{1}$, RAGHU HARSH KALIA ${ }^{2}$, MONICA KAUSHIK $^{3}$, MONIKA ARORA ${ }^{4}$}

Department of Electronics and Communication Engineering, Amity University, UP, India. Email: barkhapandey256@gmail.com¹,raghuharsh007@rocketmail.com², maushik@amity.edu ${ }^{3}$, marora@amity.edu ${ }^{4}$

Published Online: December 28, 2016

The Author(s) 2016. This article is published with open access at www.chitkara.edu.in/publications
Abstract: In a last few decades there has been tremendous growth and a drastic rise in wireless communication technologies. The major issue that the world is facing today with the advent of so many sophisticated wireless communication devices is efficient utilization of the spectrum. Spectrum efficiency comes together with a tradeoff factor of energy consumption. Thus, the need of the hour is an energy and bandwidth efficient technique. DS-CDMA is one of the competitive and most investigated technique in wireless communication which caters the large demand at the same time with limited bandwidth. Existing modulation techniques does not promise to provide better performance in case of higher noise. Therefore, one looks for a better BER stipulated modulation format to improve and enhance the performance of the system. This article revisits conventional Binary phase shift keying method and compares its performance in terms of BER with a special Extended Binary Phase Shift Keying (EBPSK) modulation format for a DSCDMA system. EBPSK scheme is flexible and simple which is easily integrated in a network and assures improved and enhanced performance.

Keywords: Additive White Gaussian Noise, Bandwidth Efficiency, Binary Phase Shift Keying, Bit Error Rate, Direct Sequence-Code Division Multiple Access, Extended BPSK, Modulation.

\section{INTRODUCTION}

Recent advancements in the field of wireless communication exhibit an exponential increase in the demand in terms of users, energy consumption as well as the frequency spectrum. The need of the hour is to alleviate the spectrum crisis and energy consumption problem. The ever increasing demand for better performance compels us to look in to the basic modulation technique [1-2]. Advancement in newer technology requires gaining maximum throughput using limited bandwidth that comes with efficient utilization of the available resources. CDMA (Code Division Multiple Access) is a communication technique that allows a number of users to access the
Journal on Today's Ideas Tomorrow's Technologies, Vol. 4, No. 2, December 2016 pp. $172-180$

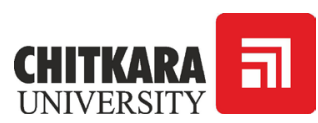


same channel simultaneously, wherein DS-CDMA (Direct sequence CDMA) is its most investigated application [3]. DS-CDMA system is a widely used communication system that faces multiple user access interference as a challenge which causes degradation in the system performance. To suffice the growing need for high data rate communication systems, a new technique which can efficiently to the work has evolved which promises to offer high spectrum efficiency and high data rates $[4,5]$. In this paper, the performance of a DSCDMA system is evaluated in terms of BER using conventional BPSK (Binary Phase Shift Keying) modulation technique, and another modulation technique referred to as extended binary phase shift keying (EBPSK) [5]. EBPSK is an ultra narrowband modulation technique which offers better energy and spectral efficiency [6]. Here, the performance of a DS-CDMA system is evaluated and simulated using BPSK modulation in terms of ratio of SNR (Signal-to-noiseratio) and BER (Bit Error Rate). Further, evaluation is done using EBPSK as a modulation technique and corresponding enhanced results are observed in presence of AWGN noise. This work sums up the advantage of taking EBPSK as a modulation technique over BPSK modulation scheme. It is known that BER for DS-CDMA system under PSK is better than ASK and FSK [7].

Section II re-considers the BPSK modulation scheme and introduces the EPBPSK modulation method and its explanation. Section III takes up BER (bit error rate) as a parameter for measuring the performance of DS-CDMA system with two different modulation formats (BPSK \& EBPSK)

\section{SYSTEM MODEL}

A DS-CDMA system is considered with 2 users using conventional BPSK modulation technique for transmission of information under AWGN environment. Generally, binary sequences are used and mutually orthogonal codes are employed for coding. This coded information is then modulated using different modulation methods. Here, we modulate the coded data with BPSK and EBPSK methods for a comparative study. As a matter of fact, BPSK uses phase variation of the carrier signal according to the input data to differentiate the bits ' 0 ' and ' 1 '. BPSK is a well known method for modulation which uses finite number of phase where each phase is allocated a special pattern of binary digits [8]. This sequence of bits forms the symbol that is depicted by the particular phase. The general form of BPSK is expressed as:

$$
s_{n}=\sqrt{\frac{2 E_{b}}{T_{b}}} \cos \left(2 \pi f_{c} t+\pi(1-n)\right), n=0,1
$$

This gives two phases, 0 and $\pi$. $f_{c}$ is the frequency of the carrier-wave. In the specific form, binary data is often conveyed with the following signals:
Performance

Enhancement of DS-CDMA System using Extended BPSK Modulation Scheme 
Pandey, B.

Kalia, R. H.

Kaushik, M.

Arora, M.

For binary " 0 "

$$
s_{0}(t)=\sqrt{\frac{2 E_{b}}{T_{b}}} \cos \left(2 \pi f_{c} t+\pi\right)=-\sqrt{\frac{2 E_{b}}{T_{b}}} \cos \left(2 \pi f_{c} t\right)
$$

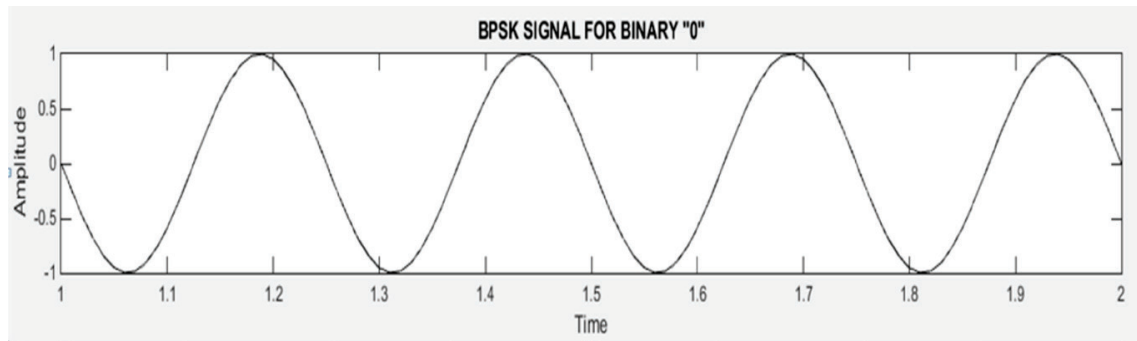

Figure 1- BPSK modulation waveform for "1"

For binary "1"

$$
s_{1}(t)=\sqrt{\frac{2 E_{b}}{T_{b}}} \cos \left(2 \pi f_{c} t\right)
$$

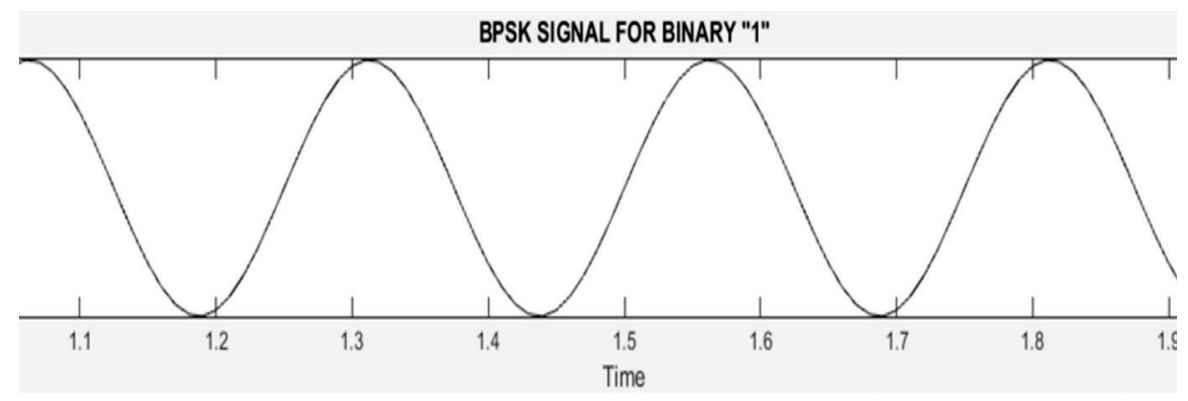

Figure 2- BPSK modulation waveform for "1"

As discussed earlier, EBPSK scheme has evolved as a new innovative UNB modulation format to achieve higher spectral, bandwidth and energy efficiency [6]. The ever increasing need for more frequency resources has compelled the researchers to look for a solution. But most of the higher level modulation techniques produce denser constellations which in turn makes the system complex and energy inefficient [8]. Extended Binary phase shift keying has thus been proposed as an energy efficient solution to the problem. The EBPSK modulation scheme can be expressed as [3]: 


$$
\begin{gathered}
\mathrm{E}_{0}(\mathrm{t})=\mathrm{A} \sin \left(2 \pi f_{c} \mathrm{t}\right), \\
E_{1}(t)=\left\{\begin{array}{cc}
B \sin \left(2 \pi f_{c} t+\theta\right) & 0 \leq \theta \leq \mathrm{t}<\mathrm{T} \\
A \sin \left(2 \pi f_{c} t\right), & 0 \leq t<\tau
\end{array}\right.
\end{gathered}
$$

where $\mathrm{E}_{0}(\mathrm{t})$ and $\mathrm{E}_{1}(\mathrm{t})$ represent digital ' 0 ' and ' 1 ' respectively. EBPSK can be viewed as flexible modulation scheme with 5 parameters namely $\theta$ (modulation index ), $\tau$ ( $\tau / \mathrm{T}$ is modulation duty ), $\mathrm{N}$ (bit cycle time), $\mathrm{K}$ (phase jump or modulation time), $\mathrm{T}$ (bit duration), $\mathrm{K}$ (phase jump or modulation time), $f_{c}$ (fixed carrier frequency). Here, $T$ can be used to manage the bit rate by either taking a short or long bit duration and obtain a higher or lower bit arte respectively. The given five parameters are related as:

$$
\begin{aligned}
& \mathrm{T}=\mathrm{N} / \mathrm{f}_{c} \\
& \tau=\mathrm{k} / \mathrm{f}_{c}
\end{aligned}
$$

There exists a very small difference in the bit " 0 " \& " 1 " waveforms [11] which makes its detection difficult. An AWGN environment is assumed so as to use a simple amplitude detector for separation of the symbols " 0 " and " 1 " $[9,10]$. The above written equation $(4,5)$ suggests that a digital ' 0 ' is represented by sine pulse and ' 1 ' with a combination of two sine pulses. If we look at digital ' 1 ' pulse we can find a phase shift of 180 degree at the start, this is because $\theta$ (modulation index) is $\pi$ in this case. Moreover, the values of A and B in the given equations $(4,5)$ which represents the amplitude remains same in this case.

The EBPSK modulated bit " 0 " and " 1 " can be represented through following waveforms:
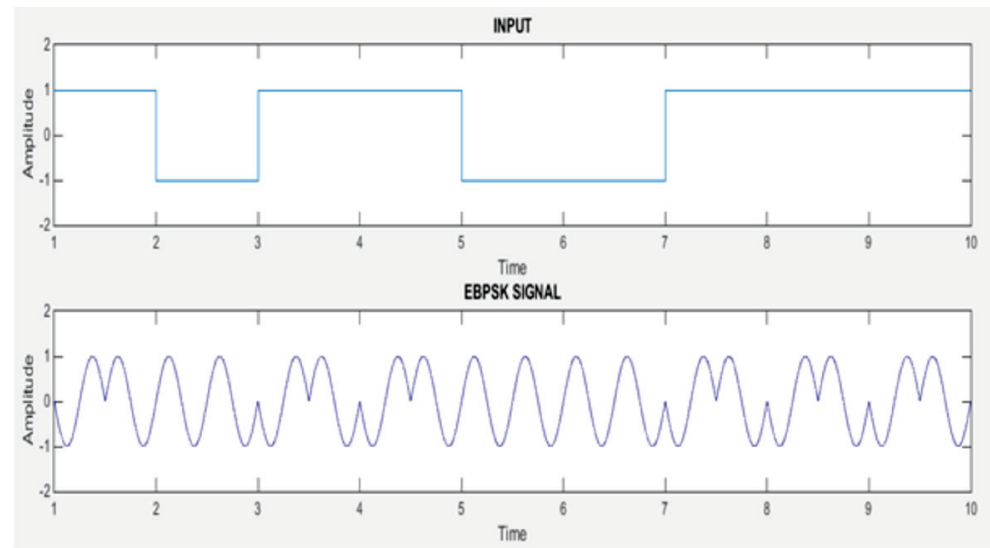

Figure 3: EBPSK Modulation at $\theta$ (modulation index $)=p$ 
Pandey, B. Kalia, R. H. Kaushik, M. Arora, M.

It is observed from EBPSK modulated waveforms that a very small angle phase and jump time is employed to distinguish bit " 1 " from bit " 0 " which results in a compressed spectrum. Thus, EBPSK modulation scheme can be regarded as an energy as well as spectrum efficient modulation method.

\section{PERFORMANCE ANALYSIS USING BIT ERROR RATE}

Bit error rate (BER) plays a vital role in measurement and analysis of performance of any communication system. A system with good BER means the modulation which is being used has reduced the effect of noise upon the modulated data. BER is calculated as number of bits in error to the actual transmitted bits. This section compares the BER for BPSK and EBPSK modulation schemes. The receiver model calculates the data with error and the plot (figure 4, 5 and 6) shows a relationship between $\mathrm{E}_{\mathrm{b}} / \mathrm{N}_{\mathrm{o}}$ and BER where $\mathrm{E}_{\mathrm{b}}$ is the signal energy linked with each user data bit and $\mathrm{N}_{\mathrm{o}}$ is the noise spectral density. Our experimental simulated results show that EBPSK outperforms the BPSK modulation scheme in terms of BER.

\section{A. Experimental Results}

In this section simulation results are given, which illustrates the feasibility of EBPSK modulation over BPSK modulation when DS-CDMA system is considered. It can be observed that when we increase the signal power $\left(\mathrm{E}_{\mathrm{b}} / \mathrm{N}_{\mathrm{o}}\right)$ the error reduces, that means better BER performance. First, BER of BPSK (figure 4) and EBPSK (figure 5)is plotted separately then comparison(figure 6) is made between the two by taking BER as percentage. Table 1 and 2 provide a deeper understanding between the two schemes used.

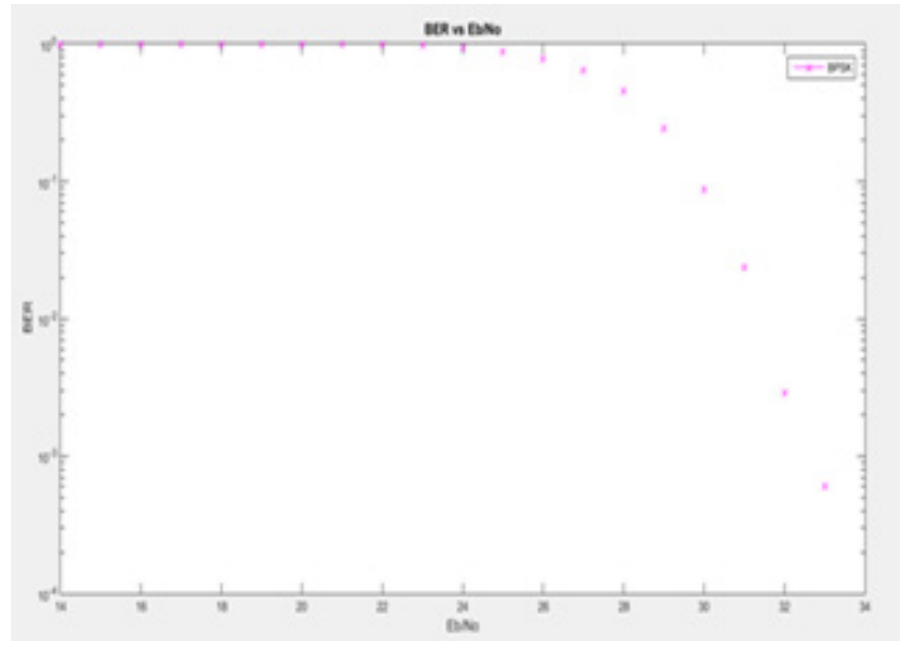

Figure 4: BER for BPSK modulation 
Table 1: Comparison of BER with different $\mathrm{E}_{\mathrm{b}} / \mathrm{N}_{\mathrm{o}}$ values for BPSK modulation

\begin{tabular}{|c|c|}
\hline $\mathrm{E}_{\mathrm{b}} / \mathrm{N}_{\mathrm{o}}$ & $\mathrm{BER}$ \\
\hline 15 & 0.9999 \\
\hline 19 & 0.9998 \\
\hline 20 & 0.9995 \\
\hline 21 & 0.9991 \\
\hline 22 & 0.9963 \\
\hline 23 & 0.9904 \\
\hline 24 & 0.9722 \\
\hline 25 & 0.9345 \\
\hline 26 & 0.8668 \\
\hline 27 & 0.7693 \\
\hline 28 & 0.6394 \\
\hline 29 & 0.4455 \\
\hline 30 & 0.2296 \\
\hline 31 & 0.0872 \\
\hline 33 & 0.0047 \\
\hline 34 & 0.0009 \\
\hline 35 & 0 \\
\hline &
\end{tabular}

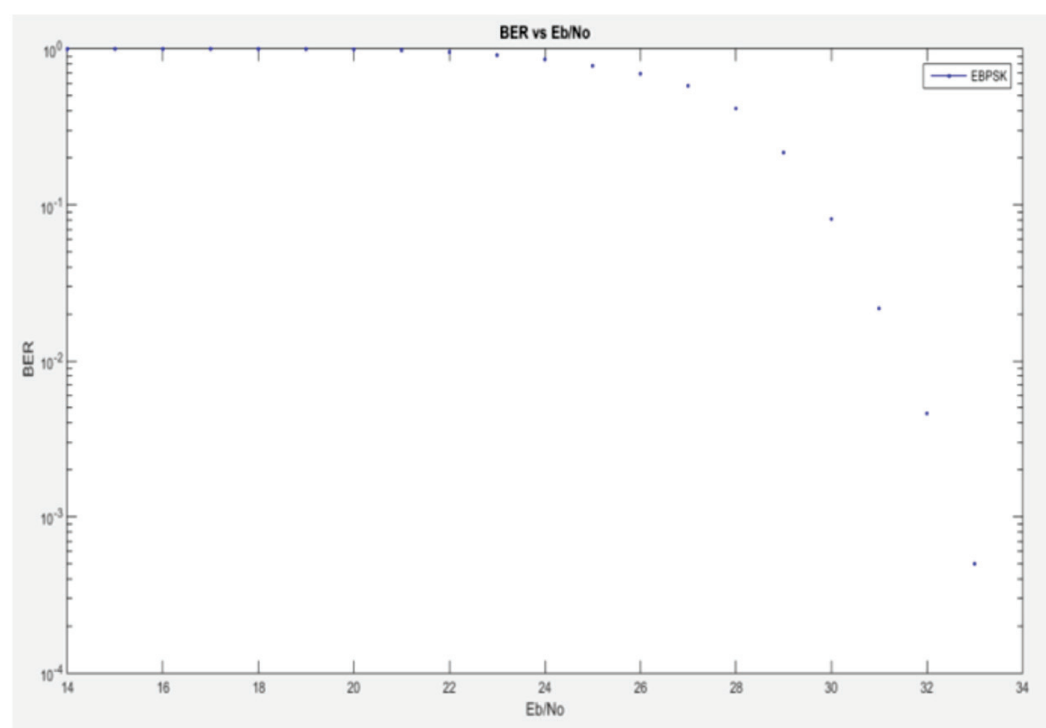

Figure 5: BER for EBPSK modulation
Performance Enhancement of DS-CDMA System using Extended BPSK Modulation Scheme 
Pandey, B.

Kalia, R. H.

Kaushik, M.

Arora, M.
Table 2: Comparison of BER with different $\mathrm{E}_{\mathrm{b}} / \mathrm{N}_{\mathrm{o}}$ values for EBPSK modulation

\begin{tabular}{|c|c|}
\hline $\mathrm{E}_{\mathrm{b}} / \mathrm{N}_{\mathrm{o}}$ & BER \\
\hline 15 & 0.9999 \\
\hline 19 & 0.9983 \\
\hline 20 & 0.9970 \\
\hline 21 & 0.9903 \\
\hline 22 & 0.9753 \\
\hline 23 & 0.9527 \\
\hline 24 & 0.9091 \\
\hline 25 & 0.8587 \\
\hline 26 & 0.7769 \\
\hline 27 & 0.6973 \\
\hline 28 & 0.5745 \\
\hline 29 & 0.4129 \\
\hline 30 & 0.2189 \\
\hline 31 & 0.0809 \\
\hline 33 & 0.0044 \\
\hline 34 & 0.0008 \\
\hline 35 & 0 \\
\hline
\end{tabular}

Figure 6 compares the BER of two schemes for different $E_{b} / N_{o}$ values. This figure gives a better understanding of the performance evaluation as the $y$ axis which represents BER is taken in percentage. In the beginning, when noise was high, the two schemes do not show a good performance. When $\mathrm{E}_{\mathrm{b}} / \mathrm{N}_{\mathrm{o}}$ value is increased then EBPSK shows remarkable progress, performance increased linearly from $4 \%$ at $23 \mathrm{db}$ to $9 \%$ at $26 \mathrm{db}$, afterwards with an increase in $\mathrm{E}_{\mathrm{b}} /$ $\mathrm{N}_{\mathrm{o}}$, performance of both schemes become almost equal but still EBPSK performance has better result than BPSK although the difference between them is not much. 


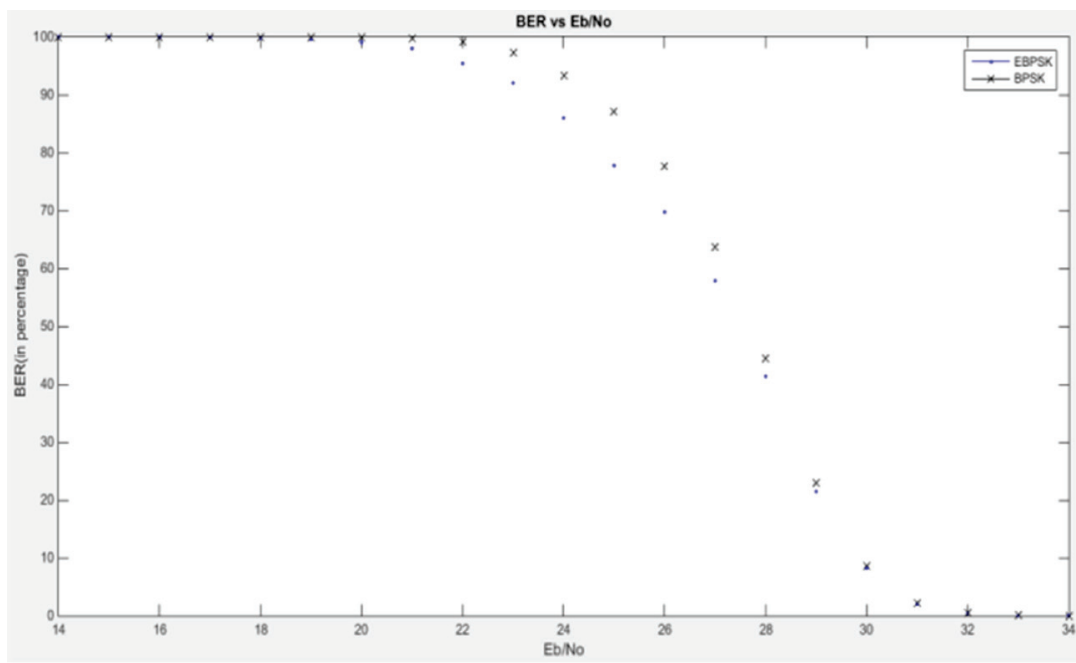

Performance Enhancement of DS-CDMA System using Extended BPSK Modulation Scheme

Figure 6: BER comparison of EBPSK and BPSK modulation

\section{CONCLUSION}

In this paper we have addressed a number of advantages of EBPSK scheme for the present day scenario of Wireless Communication. EBPSK method promises to provide lesser energy consumption and better spectral efficiency. However, this work has taken up conventional BPSK modulation scheme and compared its BER performance with special extended BPSK scheme for a DS-CDMA system. The reviewed literature and simulated results shows better performance of EBPSK scheme for a DS-CDMA system. The simulated results depict that there is a significant improvement in the BER performance at higher values of $E_{b} / N_{o}$ It is a technique which is simple to implement and provides better BER operation with few changes in representation of Bit "1" and " 0 ".

\section{REFERENCES}

1. Blume, O.; Zeller, D.; Barth, U., Approaches to energy efficient wireless access networks. In Proceedings of 4 th International Symposium on Communications, Control and Signal Processing (ISCCSP), Limassol, Cyprus, 3-5 March, 2010; pp. 1-5. http://dx.doi. org/10.1109/ISCCSP.2010.5463328.

2. Tucker, R.S. Green optical communications-Part II: Energy limitations in networks. IEEE J. Sel. Top. Quantum Electron. 2011, 17, 261-274. http://dx.doi.org/10.1109/ JSTQE.2010.2051217.

3. Mahmoud, Seedahmed S., Zahir M. Hussain, and Peter J. O'Shea. "BER Performance of DS-CDMA System Over a Frequency Selective Multipath Rayleigh Fading Channel." (2003). http://dx.doi.org/10.1109/ICEEICT.2015.7307511.

4. Bin, L.; Zheng, Z.; Weixia, Z.; Wanxin, G. Particle swarm optimization based non-coherent 
Pandey, B.

Kalia, R. H.

Kaushik, M.

Arora, M. detector for ultra-wideband radio in intensive multipath environments. EURASIP J. Adv. Signal Process. 2011, 2011, 341836. http://dx.doi.org/10.1155/2011/341836.

5. Wu, L.; Feng, M. On ber performance of ebpsk-modem in awgn channel. Sensors 2010, 10 , 3824-3834. http://dx.doi.org/10.3390/s100403824.

6. Shikai Zhang, "EBPSK Modulation with Very High Bandwidth Efficiency," International Conference on Information Science and Technology March 26-28, 2011 Nanjing, Jiangsu, China. http://dx.doi.org/10.1109/ICIST.2011.5765319.

7. M. A. Rahman, M. M. Alam, Md. Khalid Hossain, Md. Khairul Islam, Khan M. Nasir Uddin, Md. Shahinuzzaman, "Performance Evaluation of a DS-CDMA System in a Rayleigh Fading Environment", December 2015. http://dx.doi.org/10.4236/wjet.2016.41001.

8. Chen, Xian-Qing, and Le-Nan Wu. "Nonlinear detection for a high rate extended binary phase shift keying system.” Sensors 13.4 (2013): 4327-4347. http://dx.doi.org/10.3390/ s130404327.

9. S.K Zhang, L.N. Wu, "The general binary phase modulation and demodulation method," China patent Publication Number: CN1889550A, 2006.

10. Man, F.; Lenan, W.; Jiajia, D.; Chenhao, Q. "BER analysis and verification of EBPSK system in awgn channel” IEICE Trans. Commun. 2011, 94, 806-809.10.1587/transcom. E94.B.806.

11. Feng Man, Xia Shuqiang, WU Lenan, "Research on High-speed NFC Transmission Based on High-efficiency EBPSK Modulation” 2013. http://dx.doi.org/10.1049/cp.2013.2390. 